\title{
Risk-Field Based Modeling for Pedestrian Emergency Evacuation Combined with Alternative Route Strategy
}

\author{
Chao Wang and Jian Wang \\ School of Electronic and Information Engineering, Tongji University, Shanghai 201804, China \\ Correspondence should be addressed to Chao Wang; 890806wangchao@tongji.edu.cn
}

Received 20 December 2016; Revised 22 March 2017; Accepted 2 April 2017; Published 20 April 2017

Academic Editor: Antonio Bilotta

Copyright (C) 2017 Chao Wang and Jian Wang. This is an open access article distributed under the Creative Commons Attribution License, which permits unrestricted use, distribution, and reproduction in any medium, provided the original work is properly cited.

\begin{abstract}
For the past decades, with the frequent occurrence of emergencies, such as stampede, fire, and terrorist attack, the urgent requirements of developing realistic evacuation models to describe pedestrian and disaster dynamics are put forward. In this paper, an extended floor-field (FF) model combined with risk factors is presented for emergency evacuation. A novel dynamic rerouting mechanism is designed to elucidate the exit choice behavior of evacuees, and meanwhile, a recommended dynamic risk-field model is fully explored to deal with dynamical features of disaster. The proposed model is validated through numerical simulations with specific room structures. The effects of model parameters on evacuation efficiency and death toll are analyzed in detail. Simulation results show that the proposed model is effective and has a positive influence on evacuees' exit choice behavior, and the death toll is closely related to the perceived information obtained by evacuee, such as the crowd density during the evacuation process.
\end{abstract}

\section{Introduction}

In recent years, widespread concerns about effective emergency evacuation have been raised among industries and academics [1]. Rooms and buildings are the main scenarios of evacuation due to the inherent vulnerability to natural hazards and disasters such as stampede, fire, and terrorist attack [2]. During the whole evacuation process, the disaster is dynamically changing and makes lasting impacts on the decision-making of evacuees. Therefore, it is crucial to take into account the dynamical characteristics of disaster and the changing strategies for evacuees when an effective evacuation model is established.

Currently, there are two kinds of pedestrian evacuation models, that is, macroscopic and microscopic models [3]. Among the macromodels, the representative one is the fluiddynamic model $[4,5]$. In this kind of fast calculating models, pedestrians are always described with fluid attributes and usually applied to the case of large crowd without considering behaviors of individual and group. Micromodels can be further divided into continuous model and discrete model [6]. The social force (SF) model is a representative of continuous model which consists of three kinds of forces: physical force, psychological force, and social force [7]. By this means, some observed behaviors of pedestrian flow under panic circumstance can be reproduced $[7,8]$. Li et al. proposed a social force model to study earthquake evacuation that occurred in China [9]. However, SF models generally have a high computational cost which cannot meet the needs of real-time computer simulation. On the contrary, the discrete model such as the cellular automata (CA) model is discrete in topological space, time step, and state variables [10-15]. In particular, CA models have strong expressive power to represent many collective behaviors (e.g., clogging, herding behavior, friction effects, competitive egress behavior, and bidirection pedestrian behavior) with a lower computational cost and achieve very good simulation results [6]. For example, Kirchner et al. [16] proposed a modified stochastic CA model to simulate friction effects and clogging during the evacuation of a large room with one door. Yuan and Tan [17] introduced a basic CA model based on human behavior (inertial effect, group effect, and unadventurous effect) to investigate the evacuation from a smoke-filled room with multiple exits.

The floor-field (FF) model, first proposed by Burstedde et al. [10], is one of the most widely used CA models and consists 

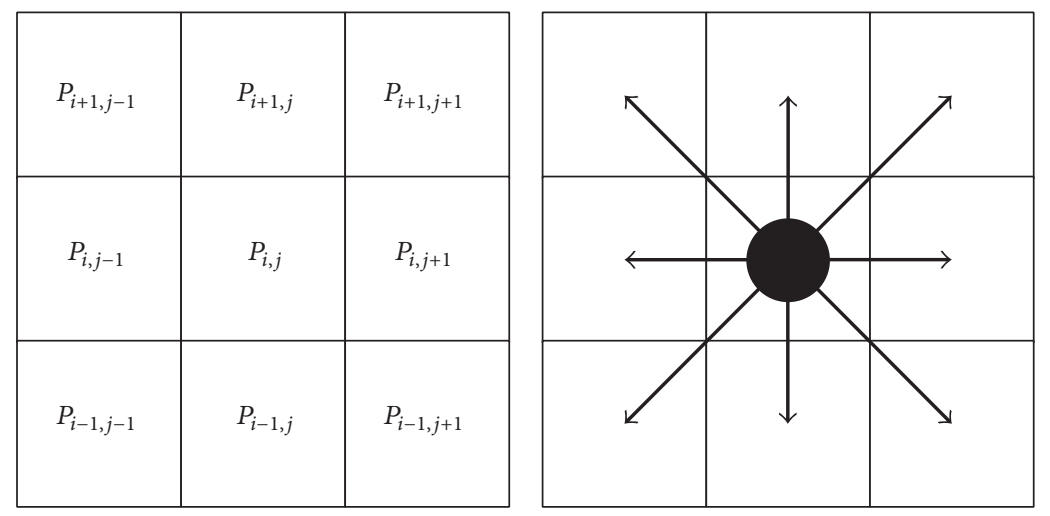

FIGURE 1: One of the eight neighboring cells can be selected as the target cells according to the transition probability $P_{i, j}$ at next time step.

of two floor fields: static-field and dynamic field. Fermion and boson are introduced into FF model to describe pedestrian's movement and the pheromone particle, respectively. Henceforth, distinct research perspectives, for example, pedestrian-pedestrian interaction [11] and pedestrian-environment interaction [12-14], are investigated to research the FF model. Of late, research efforts have been made to modify FF models. Li et al. [15] proposed a block-based floorfield model to describe the pedestrian's moving behavior of walking through corner. Huan-Huan et al. [18] proposed an improved method to calculate the floor-field, in which pedestrians are treated as movable obstacles that increase the value of the floor-field. Guo et al. [19] modified the dynamic floor-field model by introducing heterogeneous bosons. This modified model removes the confusion of traces generated by evacuee himself and exhibits realistic pedestrian behaviors. Similarly, Hartmann and Hasel [20] proposed a new computational approach based on adaptive grid concepts for the construction of dynamic floor-field to simulate realistic evacuation scenarios. Zhao and $\mathrm{Li}$ [21] introduced game theory into FF model to investigate the inertia effects on strategy updating during emergency evacuation. To be specific, game theory is adopted to deal with conflicts resolving process of evacuees and inertia effects are considered in the process of exit choice as well as conflict resolution. Lu et al. [22] extended FF model in a realistic way to describe the walking behavior of pedestrian groups. Besides, the shape of the cell is a research hotspot as well. The square cell is most commonly used in FF model while the rhombus cell [23] and the regular hexagonal cell $[24,25]$ also arouse the attention of researchers.

Some scholars have introduced the static attributes of disaster to study the evacuation process in public area [26, 27]. However, the static risk-field is inaccurate to describe the diffusion and decay features; moreover, the evacuation plans of evacuees are formulated beforehand and are not altered with the perceived scene information in real-time. In our previous work [28, 29], a modified FF model was proposed to investigate the effect of guiding information during evacuation preliminarily. In addition, we extended the model based on static risk-field to reveal the behaviors of pedestrian under emergency. In this paper, we make further improvement on our previous method, where the static riskfield is replaced by a dynamic one. Besides, eight directions are applied to depict the navigation options of pedestrians as shown in Figure 1 and the rerouting mechanism is newly introduced to establish our dynamic FF model.

The remainder of this paper is organized as follows. Section 2 sheds light on the modified FF model by considering the dynamic risk-field and rerouting mechanism. Section 3 elucidates the specific scenarios for simulation and the corresponding model parameters. Section 4 illustrates the experimental results. Section 5 presents conclusions and discusses some potential future research.

\section{Model Description}

The classical FF model usually takes into account static-field and dynamic field to calculate the transition probabilities of target cells in next time step. However, in reality, we have to consider dynamical features of disaster during emergency evacuation process. Moreover, it is common that evacuee is unfamiliar with the evacuation space, and some exits may be utterly closed once disaster occurred. Therefore, it is essential to take account of dynamical features of disaster and rerouting mechanism when calculating the transition probability.

2.1. Introduction of the Floor-Field. The floor-field (FF) model consists of two fields: the static-field $S_{i, j}$ and the dynamic field $D_{i, j} . S_{i, j}$ depicts the distance from cell $(i, j)$ to exit $\left(i^{\prime}, j^{\prime}\right)$ and $D_{i, j}$ is initially introduced to translate a long-ranged spatial interaction into an attractive local interaction [30]. In FF model, the movement of each pedestrian is decided by a transition probability algorithm $\left(P_{i, j}\right)$ which is defined as

$$
P_{i, j}=N \cdot \xi_{i, j}\left(1-\eta_{i, j}\right) \exp \left(K_{S} \cdot S_{i, j}+K_{D} \cdot D_{i, j}\right),
$$

where $N$ is the normalizing parameter for ensuring $\sum P_{i, j}=1$. $K_{S} \in[0,+\infty]$ is a weighting parameter of $S_{i, j}$ and is introduced to describe the tendency of pedestrians to evacuate along the shortest route. $K_{D} \in[0,+\infty]$ is introduced for scaling $D_{i, j}$ and is used to reflect the willingness to follow the crowd around. If cell $(i, j)$ is occupied by a pedestrian, $\xi_{i, j}=0$; 
otherwise, $\xi_{i, j}=1$. If cell $(i, j)$ is occupied with fixed obstacle or disaster $\eta_{i, j}=1$; otherwise, $\eta_{i, j}=0$.

2.2. Rules of Static-Field. According to the geometry of the room and door location, each cell got a constant value representing its distance to the door and this value is defined as $S_{i, j}^{\text {static }}$. The rules of calculating $S_{i, j}^{\text {static }}$ are denoted as follows:

(1) The room is discretized into several rectangular grids. The location of exit is defined as " $S_{i, j}^{\text {static }}=0$."

(2) Then the "second layer" of cells adjacent to the exits are assigned a value, according to the following rules:

(2.1) if a cell has value " $N$," the adjacent cells in the vertical or horizontal direction and in diagonal directions are assigned a value of " $N+1$ " and " $N+1.5$," respectively,

(2.2) if there are conflicts in the value assignment, the minimum possible value is assigned to this cell.

(3) The process is repeated till all cells are evaluated.

(4) Cells representing walls and obstacles are given a fixed value of 1000, which ensures that pedestrians will never attempt to occupy these cells.

In this research, basic principles for pedestrian movements and collision-avoiding are based on the following rules:

(5) Each pedestrian can move one cell in each time step based on the transition probability algorithm (the cell with the largest probability is chosen as the target).

(6) If more than one evacuee has the same target cell, randomly assign it to one of them and then the others stay in the original cells.

2.3. Rules of Exit-Field. In this paper, we introduce a new parameter $S_{i}^{\text {static }}(i \in[1,2, \ldots, n], n$ is the number of exits) and we call it exit-field. For example, $S_{1}^{\text {static }}$ represents the field value calculated based on exit 1 and, at this point, the rest exits are regarded as inexistence. More importantly, the new parameter $S_{i}^{\text {static }}$ reflects the behavior that evacuees are willing to quickly leave from the room through the nearest exit when accident just happens. The value of $S_{i}^{\text {static }}$ is calculated according to the rules of static-field.

2.4. Algorithm of Dynamic Field. Kirchner and Schadschneider [31] proposed a dynamic floor-field model which decays with a probability and diffuses with some probability to one of its four neighbor cells. In this research, we extend the dynamic floor-field model to eight neighbor cells and the value of $D_{i, j}^{t}$ is abstracted as the willingness to follow other evacuees. The value of dynamic floor-field is calculated according to the following principles:

(1) At $t=0$ time step, the dynamic field is zero; that is, $D_{i, j}=0$. Whenever a pedestrian jumps from cell $(i, j)$ to one of the neighboring cells, $D_{i, j}$ at the original cell is increased by one. More specifically, whenever a person leaves from cell $(i, j)$ to $(x, y)$, he drops a boson at cell $(i, j)$; that is, $D_{i, j} \rightarrow D_{i, j}+1$, and the value of $D_{i, j}$ at cell $(i, j)$ is the number of bosons.

(2) The boson has its own dynamics, namely, diffusion and decay, which leads to broadening, dilution, and finally vanishing of the trace [31]. In every time step, the bosons of cell $(i, j)$ diffuse and decay with probabilities $\alpha$ and $\delta$, respectively, so $D_{i, j}=D_{i, j}(t, \alpha, \delta)$. More specifically, the diffusion and decay process can be written as follows:

\section{Diffusion process is}

$$
D_{i, j}^{t+1}=D_{i, j}^{t+0.5}-\alpha D_{i, j}^{t+0.5}+\frac{\alpha}{8}\left(\sum_{i}^{i \pm 1} \sum_{j}^{j \pm 1} D_{i, j}^{t+0.5}-D_{i, j}^{t+0.5}\right) .
$$

Decay process is

$$
D_{i, j}^{t+0.5}=D_{i, j}^{t}-\delta D_{i, j}^{t}
$$

The dynamic floor-field after combination is

$$
\begin{aligned}
D_{i, j}^{t+1}= & (1-\alpha)(1-\delta) D_{i, j}^{t} \\
& +\frac{\alpha(1-\delta)}{8}\left(\sum_{i}^{i \pm 1} \sum_{j}^{j \pm 1} D_{i, j}^{t}-D_{i, j}^{t}\right) .
\end{aligned}
$$

2.5. Rules of Dynamic Risk-Field. In the real world, a majority of disasters are dynamic evolution processes with immanent mechanisms. In the research of Han et al. [32], an integrated real-time evacuation route planning method was proposed and was composed of real-time data acquisition, risk distribution calculation, and evacuation route formulation. Leng et al. [25] proposed an extended floor-field model which takes into account the effect of wall repulsion and pedestrian repulsion instead of considering any kinds of fatalities. In addition, treating the accident as a fixed obstacle is also researched by scholars [33]. In this research, we introduce a dynamic risk-field $\left(R_{i, j}\right)$ to depict the dynamic features of disaster, for example, fire or toxic gas. The value of $R_{i, j}$ is calculated according to the following rules:

(1) The value of dynamic risk-field is " 0 " until the disaster occurs.

(2) We introduce two new concepts "disaster area" and "disaster influential area" formulated as $S^{*}$ and $\Gamma^{*}$, respectively. $S^{*} \propto\left(i^{*} \pm r, j^{*} \pm r\right)$ represents the area that is occupied by disaster and $\Gamma^{*} \propto\left(i^{*} \pm r^{\prime}, j^{*} \pm r^{\prime}\right)$ represents the area influenced by disaster; $r^{\prime}$ is the radius of $\Gamma^{*}$, as shown in Figure 2 . Here $\left(i^{*}, j^{*}\right)$ is the coordinate of disaster point and $r$ is the radius of $S^{*}$ which is calculated as

$$
r=\left[\frac{v \cdot t}{0.4}\right]
$$

where "[ ]" means rounding and $v$ is the spread rate of disaster. Constant value 0.4 represents the length of each cell. 


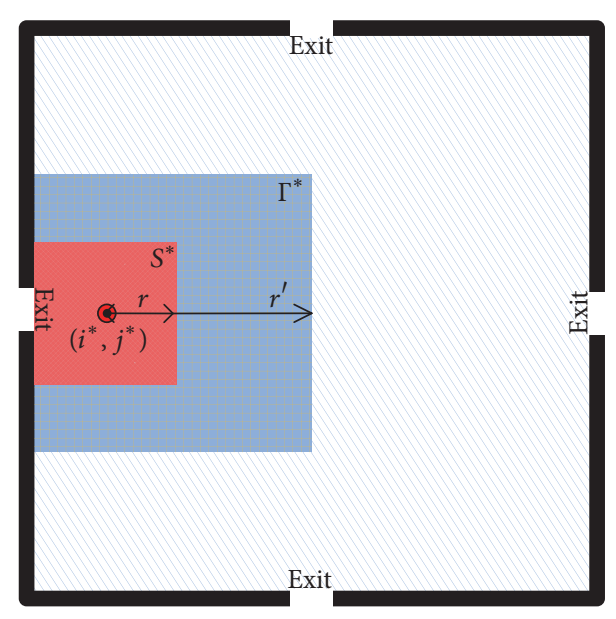

FIGURE 2: The red and blue regions represent $S^{*}$ and $\Gamma^{*}$, respectively. The grey area with oblique lines shows the area that is not influenced by the dynamic disaster.

(3) The dynamic risk-field is calculated as follows:

$$
\begin{aligned}
& R_{i, j} \\
& = \begin{cases}0, & \text { if }(i, j) \notin \Gamma^{*} \\
\frac{1}{\sqrt{\min \left\{\left(i-i^{*}\right)^{2}+\left(j-j^{S^{*}}\right)^{2}\right\}}}+1, & \text { if }(i, j) \in \Gamma^{*} \\
2, & \text { if }(i, j) \in S^{*},\end{cases}
\end{aligned}
$$

where $(i, j)$ and $\left(i^{S^{*}}, j^{S^{*}}\right)$ represent the coordinates of evacuee and the outermost cells of $S^{*}$, respectively. More specifically, $\sqrt{\min \left\{\left(i-i^{S^{*}}\right)^{2}+\left(j-j^{S^{*}}\right)^{2}\right\}}$ symbolizes the minimum distance between pedestrian $(i, j)$ and $S^{*} \cdot R_{i, j}=1 / \sqrt{\min \left\{\left(i-i^{S^{*}}\right)^{2}+\left(j-j^{S^{*}}\right)^{2}\right\}}+1$ indicates a trend that pedestrians nearby $S^{*}$ obtain a larger $R_{i, j}$, and similarly, those who are far from $S^{*}$ obtain a relatively smaller $R_{i, j}$.

2.6. Route-Choice Mechanism. In previous research, the transition probability of pedestrian is decided by the interaction of static floor-field and dynamic floor-field. The transition probability $P_{x, y}$ of a pedestrian $i$ at time $t$ moving from cell $(i, j)$ to cell $(x, y)((x, y) \neq(i, j))$ is calculated according to formula (1) during the whole evacuation process. However, in a real-life scenario, not all evacuees are familiar with the evacuation space and they cannot know where the emergency happens at first. When evacuees decide to escape from the inside, an evacuation route is predetermined. Generally speaking, evacuees close to the accident point will endeavor to flee from the hazard. Besides, those who are not familiar with the accident will not change the predetermined evacuation route until the accident is perceived. In order to build a more realistic model, we introduce a new parameter $\zeta \epsilon$ $[0,+\infty)$ to describe the perception range of pedestrian. With the introduction of $\zeta$ into transition probability, a dynamic route-choice mechanism can be realized according to the following rules:

(1) At the beginning, if $\zeta<r^{*}$, the transition probability is calculated as

$$
\begin{aligned}
P_{x, y}= & N \cdot \xi_{x, y}\left(1-\eta_{x, y}\right) \\
& \cdot \exp \left[K_{S} \cdot S_{x, y}^{\text {static }}+K_{D} \cdot D_{x, y}^{t}\right],
\end{aligned}
$$

where $r^{*}$ is the distance between evacuee $i$ and the border of $S^{*}$.

(2) When the evacuees get enough evacuation information, namely, $\zeta \geq r^{*}$, they will give up their original path and the substituted navigation strategy is executed according to

$$
\begin{aligned}
P_{x, y}= & N \cdot \xi_{x, y}\left(1-\eta_{x, y}\right) \\
& \cdot \exp \left[K_{S} \cdot S_{i}^{*}+K_{D} \cdot D_{x, y}^{t}+K_{R} \cdot R_{x, y}\right],
\end{aligned}
$$

where $S_{i}^{*}$ represents the adjusted static-field and is calculated without considering the preselected exit. This is because, by instinct, pedestrians tend to avoid the hazard and choose a new path under panic circumstance; see Figure 3.

\section{Scenarios and Model Parameters}

A room with size of $12 \mathrm{~m} \times 12 \mathrm{~m}$ (30 cells $\times 30$ cells) is investigated in this paper which is the same as our previous research [25] and the degree of discretization is $0.4 \mathrm{~m} \times 0.4 \mathrm{~m}$ for each cell. During initialization, pedestrians are allocated to cells at random. Figure 4 shows diverse exit locations in different experiment scenarios; more specifically, Figure 4(a) depicts a symmetrical structure in horizontal and vertical directions and exits are situated in each wall; Figure 4(b) represents a symmetrical structure in vertical direction and exits are situated in the left and right; and Figure 4(c) describes a symmetrical structure in horizontal direction and all exits are situated in the left.

On the basis of the three room structures in Figure 4, a group of numerical simulations is conducted and the results are discussed as below. The model parameters are dynamically adjusted based on practical demands and the details for different simulation scenarios are shown in Table 1 . We take the averaged value by using repeated tests with each experiment repeated for 1000 runs. It is assumed that when disaster spreads to the cell occupied by a person, he/she would be dead immediately.

\section{Simulation and Results}

4.1. Evacuation Efficiency with Consideration of Dynamic RiskField. Firstly, the effects of dynamic risk-field on evacuation time with crowd density varied in different scenarios are explored elaborately, as shown in Figure 4, and the results are compared with the static model where disaster is taken as 


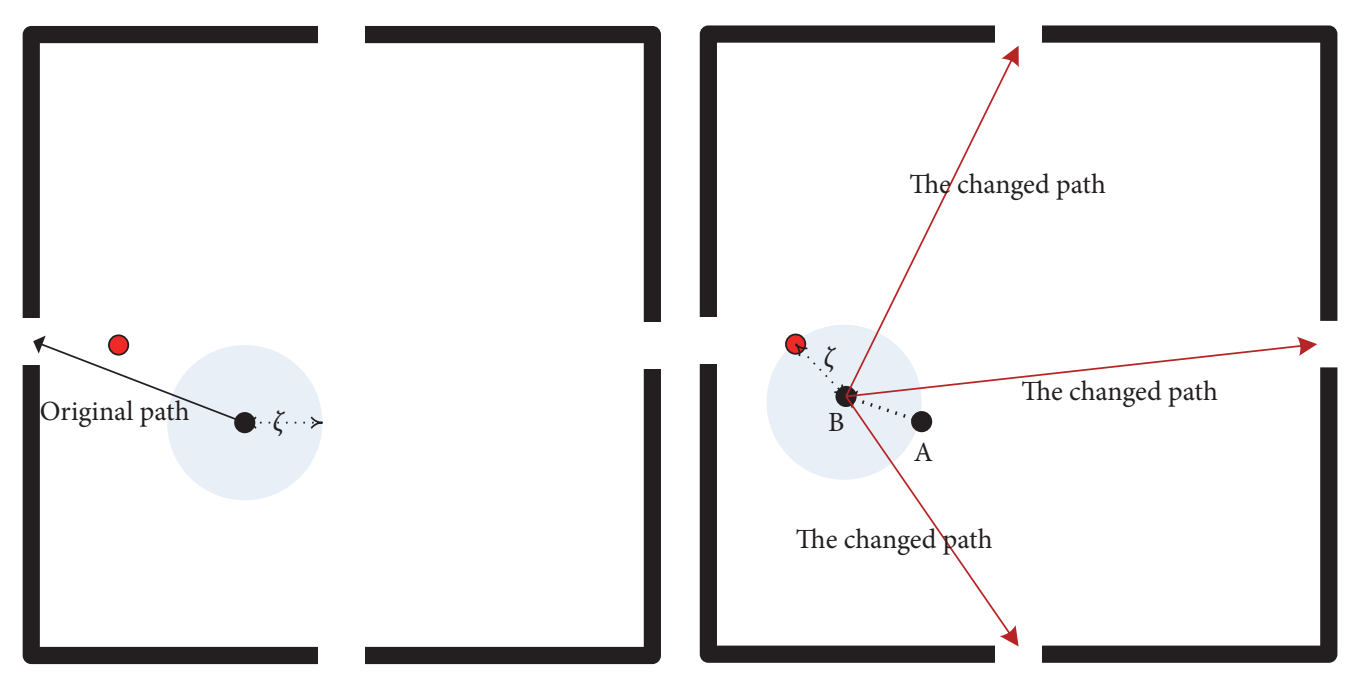

(a)

(b)

FiguRE 3: Sketch of the proposed route-choice mechanism. The black and red circles represent the disaster and pedestrian, respectively. The light blue circle is abstracted to describe the range of parameter $\zeta$. (a) The original state of evacuation with a preselected route for each pedestrian; (b) when pedestrian $i$ moves from $A$ to $\mathrm{B}$, he obtains the information about disaster and chooses a new path instead of the original path according to the rerouting principles.

TABLE 1: Model parameters.

\begin{tabular}{|c|c|c|c|c|c|c|c|c|}
\hline \multirow{2}{*}{ Section } & \multicolumn{8}{|c|}{ Parameters } \\
\hline & $\alpha$ & $\delta$ & $K_{S}$ & $K_{D}$ & $K_{R}$ & $\zeta$ & $v(\mathrm{~m} / \mathrm{s})$ & $n$ \\
\hline 3.2 .1 & 0.2 & 0.2 & 2 & 2 & 10 & 5 & 0.01 & $100-600$ \\
\hline 3.2 .2 & 0.2 & 0.2 & 2 & 2 & 10 & 6 & {$[0,0.3] ;[0,1]$} & $300 ; 100-600$ \\
\hline 3.2 .3 & 0.2 & 0.2 & 2 & 2 & 10 & {$[1,20]$} & 0.03 & 300 \\
\hline
\end{tabular}

a fixed obstacle. In this part, the dynamic risk-field situation and the static disaster situation are, respectively, recorded with solid line and dash line. The parameters are set as Table 1 shows.

We notice from Figure 5 that the evacuation time increases with the raise of pedestrian number for both the proposed dynamic model and the static model. It can also be observed that there are differences between the two models. In static model, disaster location has few influences upon the evacuation efficiency, while, in dynamic model, the effects of disaster position on evacuation efficiency are obvious. The reason for the differences is analyzed and discussed in the following paragraph.

According to Figure 5(a), the total evacuation time is almost the same when disaster occurs in the left and right; besides, the evacuation efficiency is the highest when disaster occurs in the middle of the room. This is mainly because when disaster occurs near the exits, dynamic risk-field affects the evacuees' behavior on route selecting thereby increasing the path length of those who first choose the left or the right exit as their targets. But when disaster occurs in the middle, due to the bilateral symmetrical room structure, evacuees' preselected evacuation routes will not be easily obstructed by disaster; therefore, the evacuation efficiency is the highest in this case.
As Figure 5(b) shows, whether the disaster occurs in the left or right, the evacuation process consumes as much time as each other in distribution (B). It is chiefly caused by the bilaterally symmetric exit structure and a relative low disaster spread rate. Furthermore, owing to the random distribution of evacuees, disaster occurring in which side seems to have no impact on evacuation efficiency in this specific situation. However, disaster occurring in the middle differs from the situation aforementioned. It is obvious that this circumstance achieves the best efficiency, especially for high crowd density. It also indicates that disaster occuring in the middle has less effect on evacuees' behavior in choosing exit.

However, the situation changes in Figure 5(c). Since the four exits are all situated in the left wall, it is not hard to understand that disaster occuring in the left consumes the longest evacuation time. And meanwhile, the right disaster position condition has the highest evacuation efficiency. The performance of middle disaster situation is much better than the left one but worse than the right one. It also can be illustrated that, in this specific room structure, the longer the distance between exit and disaster position, the less the time that evacuees need to spend for evacuation.

4.2. Effects of Disaster Spread Rate and Crowd Density. In this part, the impacts of disaster spread rate and crowd density 


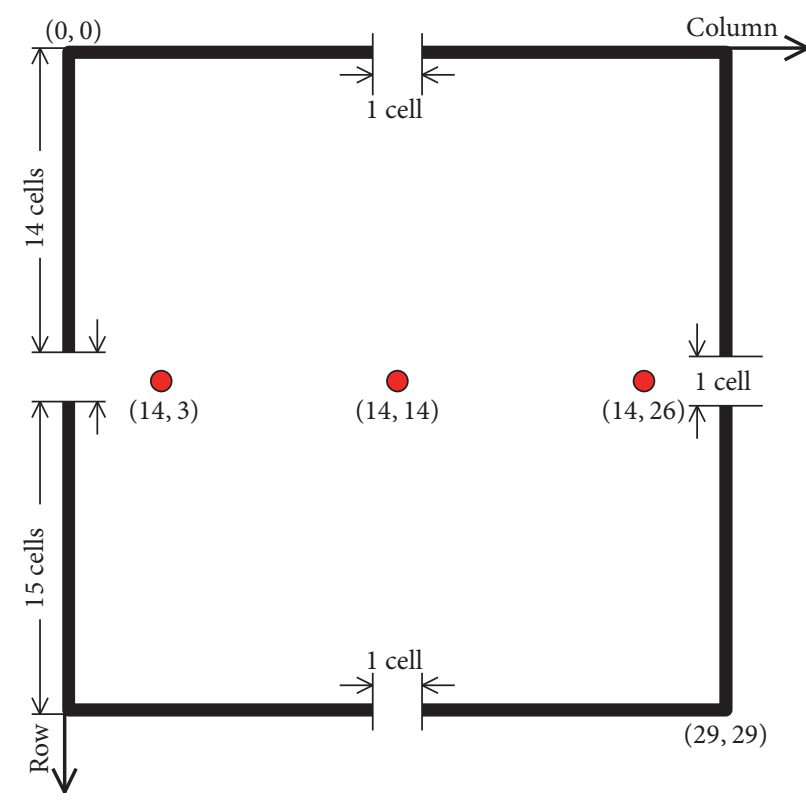

(a) Distribution (A)

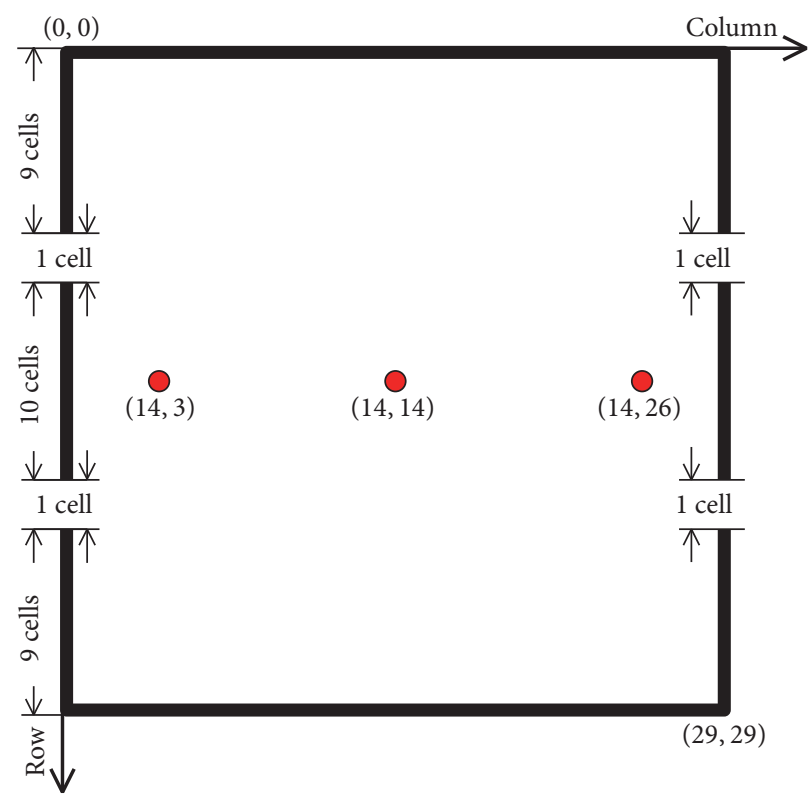

(b) Distribution (B)

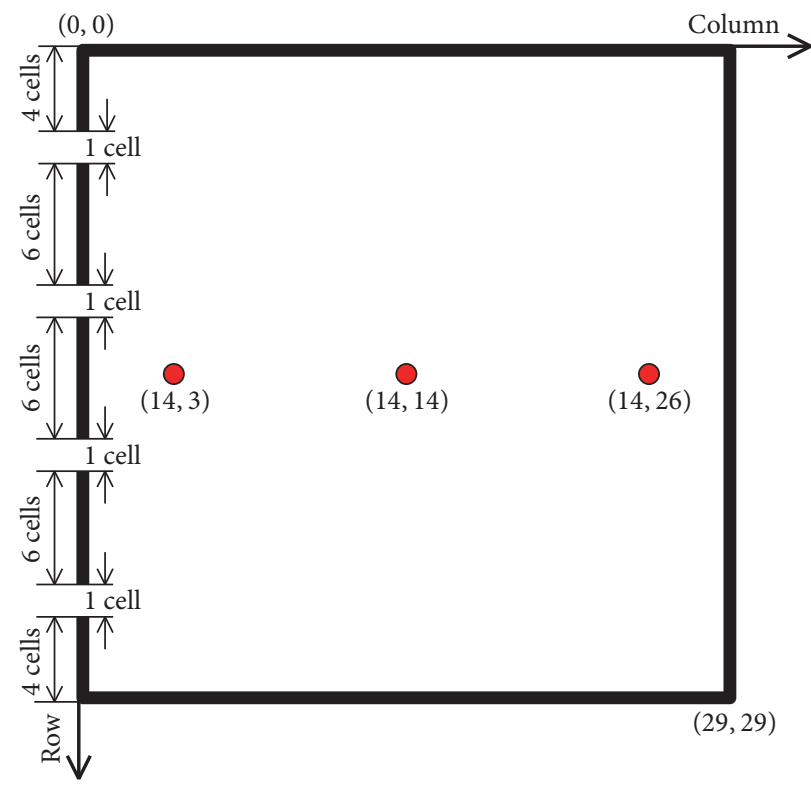

(c) Distribution (C)

FIGURE 4: Specific scenarios for evacuation simulation. The red circles represent the disaster location and the coordinate values of the entire space are labeled from 0 to 29.

on the whole evacuation process will be discussed through a series of numerical simulations. The parameters are set as Table 1 shows and the coordinate of disaster location is $(14,3)$. The effect of disaster spread rate on the death toll is shown in Figure 6.

Figure 6(a) describes the tendency of death toll with the increase of $v$ in three different layouts. It shows a significant upward trend when the spread rate increases from $0 \mathrm{~m} / \mathrm{s}$ to $0.3 \mathrm{~m} / \mathrm{s}$ and this trend tends to flat when the diffusion velocity exceeds $0.3 \mathrm{~m} / \mathrm{s}$. In view of this, we analyze the performance of three different layouts in respect of mortality when disaster spreads in its sensitive range of
$[0,0.3]$, as shown in Figure 6(b). It can be seen that the number of deaths in distribution (C) increases the fastest. In addition, distribution (A) has the minimum amount of dead people when the fire spread rate changes from $0.01 \mathrm{~m} / \mathrm{s}$ to $0.08 \mathrm{~m} / \mathrm{s}$, and after that, distribution (B) performs better than distribution (A) does. Based on these conclusions mentioned above, we implement another group of simulations to explore the correlativity between crowd density and death toll. The disaster spread rates are set as $v=0.03 \mathrm{~m} / \mathrm{s}$ and $v=0.09 \mathrm{~m} / \mathrm{s}$.

Figure 7(a) shows the trend of death toll when the number of evacuees increases with a relative low disaster spread rate. We can see from Figure 7(a) that distribution (A) has 


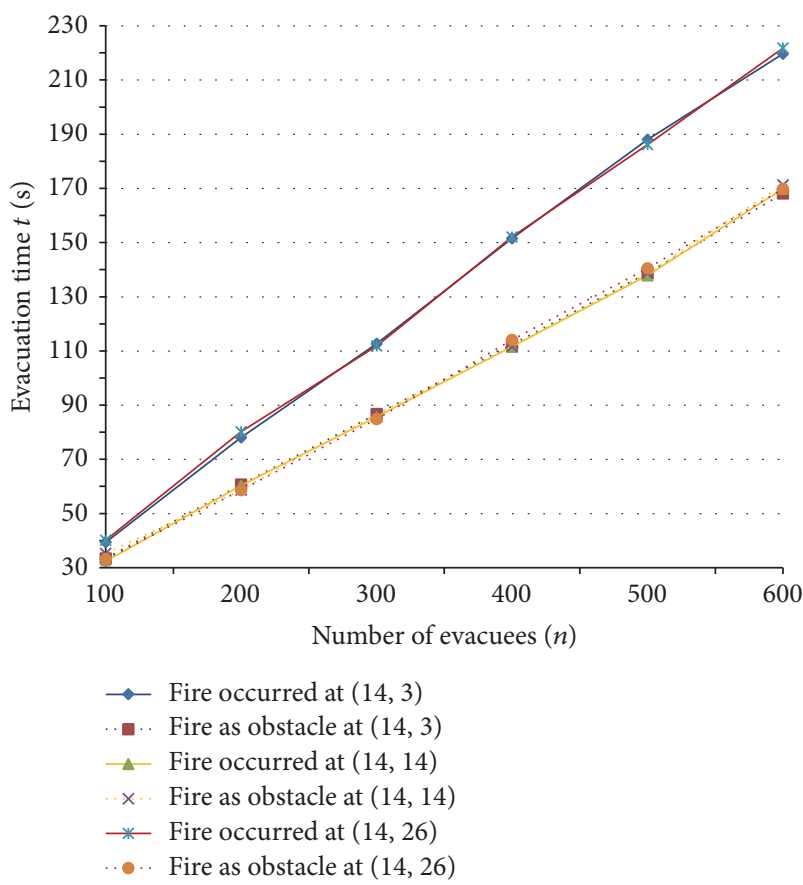

(a)

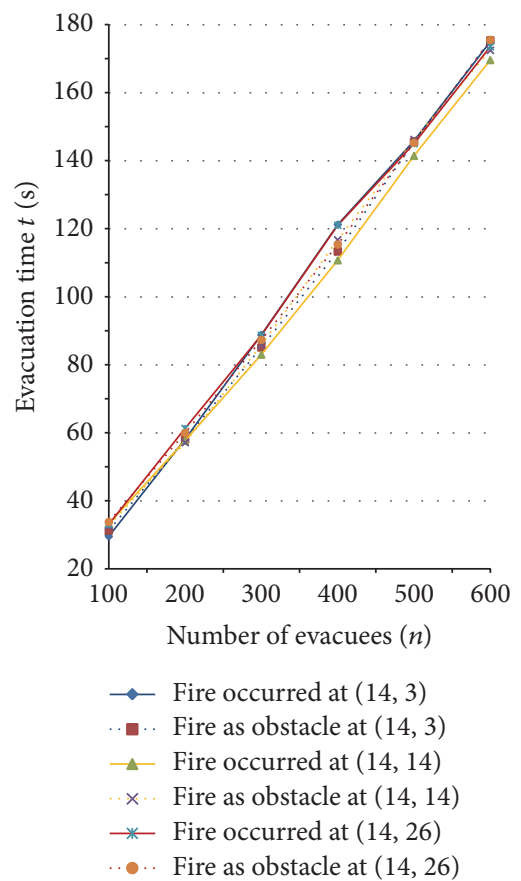

(b)

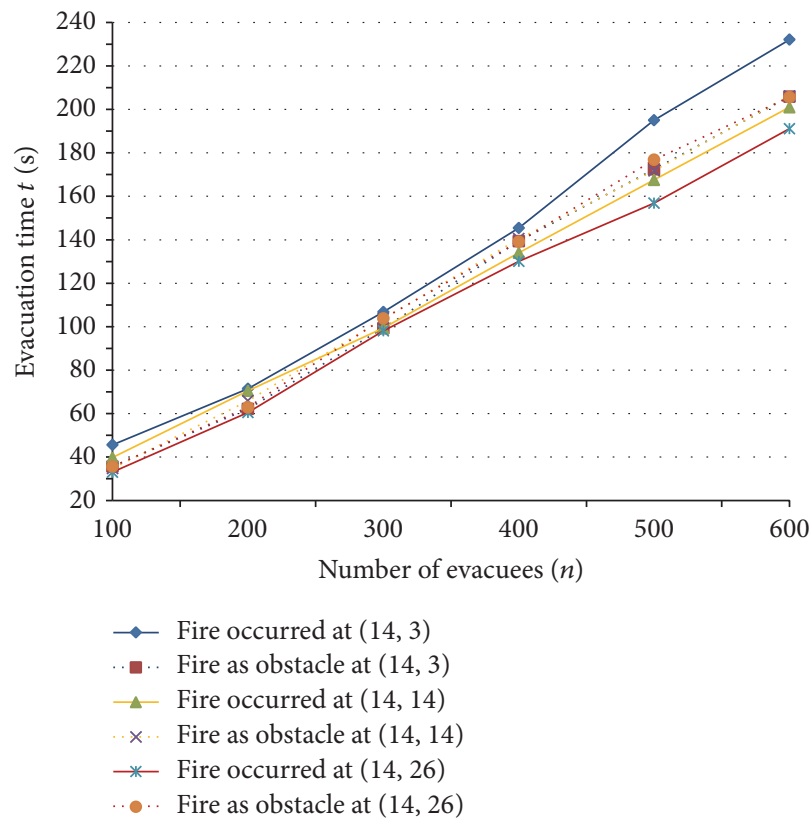

(c)

FIGURE 5: Relationship between the evacuation time and the number of evacuees with different fire positions, for example, on the left $(14,3)$, in the middle $(14,14)$, and on the right $(14,26)$ of the room. (a) For distribution (A); (b) for distribution (B); (c) for distribution (C).

the best density tolerance; in other words, the number of evacuees has limited effects on the death toll in this case. Besides, distribution (A) performs better with low pedestrian density but worse in high pedestrian density situation than distribution (B) does. Experiment results of distribution (C) are quite different from those two scenarios aforementioned. As the number of evacuees grows, especially in interval of
$[300,600]$, the death toll sharply increases due to congestion emerged near the exits.

As shown in Figure 7(b), results are different when disaster spread rate increases to a relative high value as $0.09 \mathrm{~m} / \mathrm{s}$. Distribution (B) has the lowest death rate and the death toll of distribution $(\mathrm{A})$ is always smaller than that of distribution (C). In summary, distribution (A) performs 


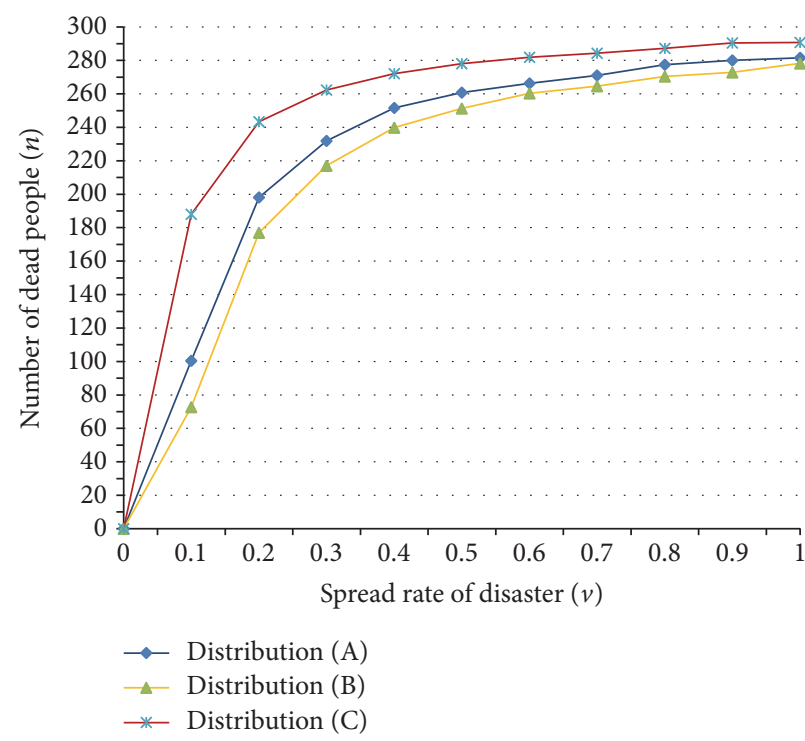

(a)

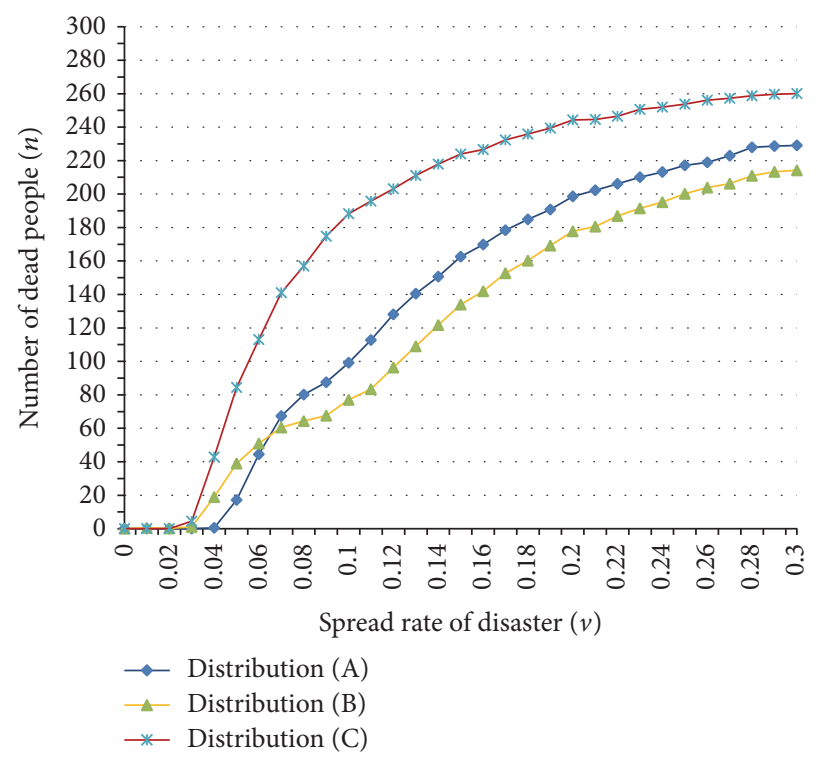

(b)

FIGURE 6: The relationship between the number of dead people $n$ and the spread rate of disaster $v$ and the disaster occurs at $(14,3)$. (a) $v \in[0,1] ; \Delta=0.1 \mathrm{~m} / \mathrm{s} ;$ (b) $v \in[0,0.3] ; \Delta=0.01 \mathrm{~m} / \mathrm{s}$.

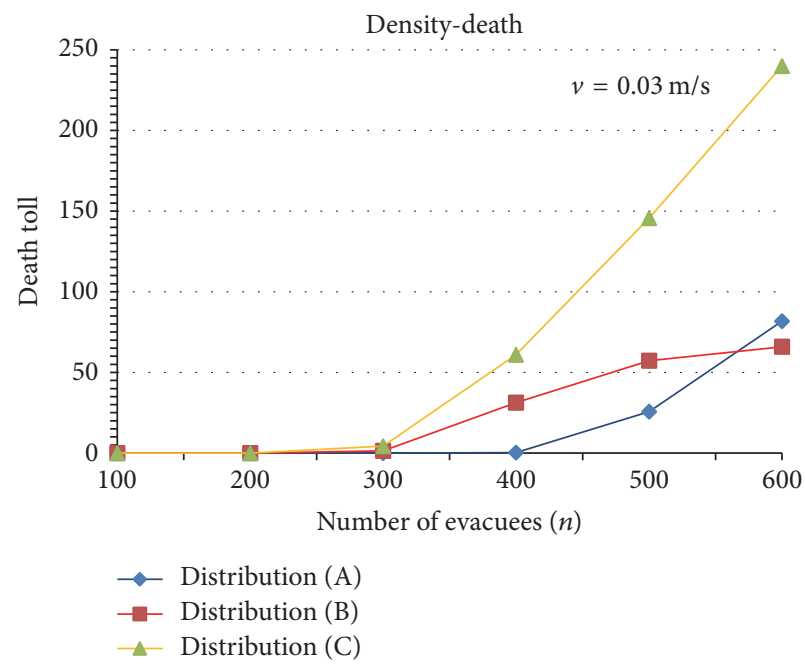

(a)

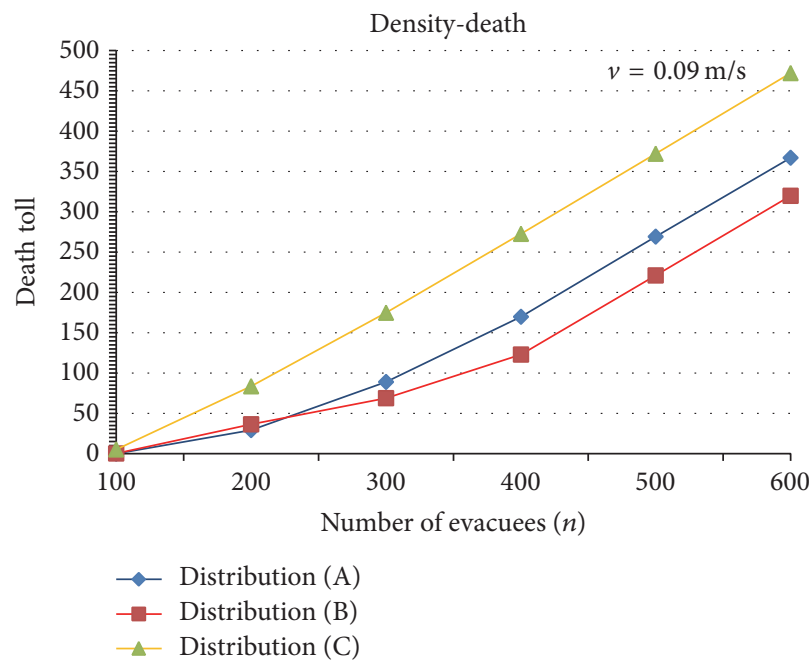

(b)

Figure 7: Correlativity between crowd density and death toll, the fire location is $(14,3)$. (a) $v=0.03 \mathrm{~m} / \mathrm{s}$; (b) $v=0.09 \mathrm{~m} / \mathrm{s}$.

better in the case that both disaster spread rate and crowd density are low while distribution (B) is more suitable for the condition with high density and spread rate. Besides, distribution $(\mathrm{C})$ is the most disappointing in high density situation when disaster occurs in the left.

4.3. Effects of $\zeta$ on Death Toll and Total Time Steps. In this section, the concept of evacuation information is introduced and a new parameter $\zeta \in[0,+\infty)$ is applied to represent the perception range of evacuees. Since parameter $\zeta$ is a key factor to affect the rerouting strategy, a group of numerical simulations is performed to evaluate the effect of $\zeta$ on evacuation process.

As Figure 8(a) shows, there is an inverse proportional relationship between parameter $\zeta$ and the death toll. Particularly, for distribution (A), the number of dead people reduces to zero when $\zeta \geq 4$. We can also observe from Figure $8(\mathrm{~b})$ that the solution of distribution (A) has a lower death rate but at the cost of more evacuation time in comparison with the solution of distribution (B). It indicates that the proposed rerouting mechanism is an effective method in evacuation despite an increase of the total distance of evacuees. In addition, the emerging congestion of exits brings about a raise 


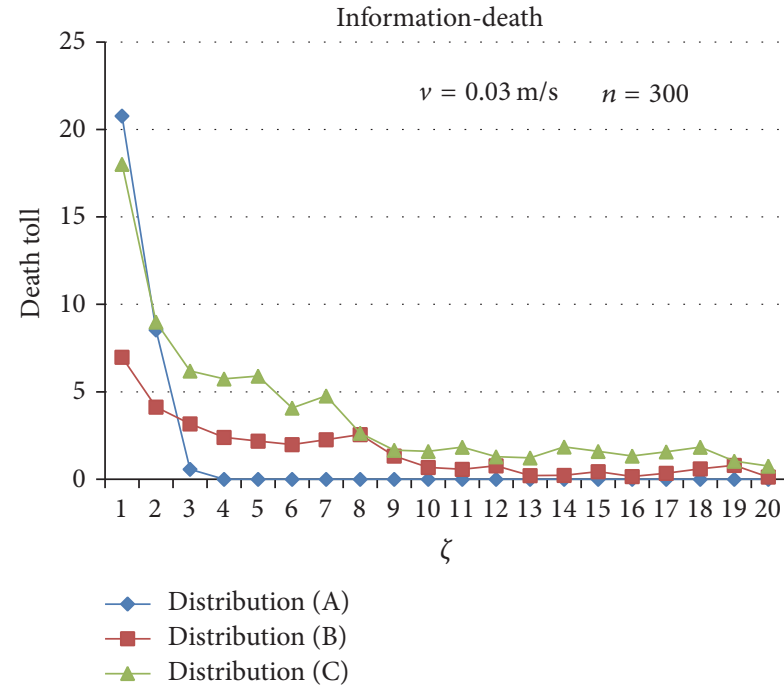

(a)

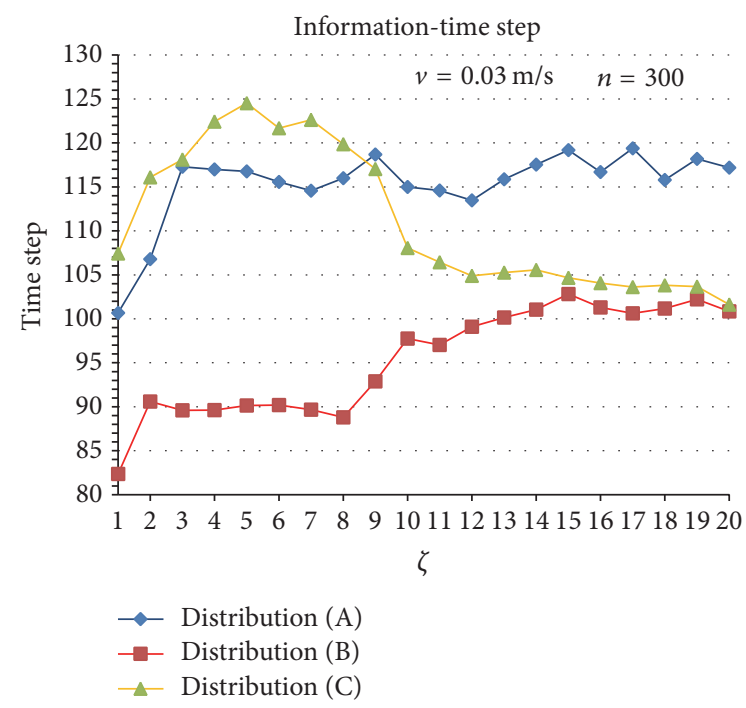

(b)

Figure 8: (a) Correlativity between parameter $\zeta$ and death toll with 300 evacuees, the disaster location is $(14,3)$. (b) The effect of parameter $\zeta$ on total time steps with 300 evacuees, the disaster location is $(14,3)$ as well.

of evacuation time as well. In other words, giving up the preselected exits could lead to congestion problems to the rest of the exits, and the congestion will be severe if the value of $\zeta$ arises.

\section{Conclusions}

In this paper, we propose an risk-field based model to depict the effect of disaster dynamics on evacuation process and introduce the dynamic route-choice mechanism to redefine the transition probability equation. Based on numerical simulations and analysis, we imitate the evacuation process and discuss the effects of main parameters. Firstly we research the correlativity between disaster position and total evacuation time with three different room structures. Then the effects of disaster spread rate and pedestrian density on total death toll are studied. At last, the roles of evacuation information and route-choice mechanism are discussed. The results show that different disaster location for specific room structures will bring diverse impacts on evacuation process. Specifically speaking, first, the higher the disaster spread rate the more the evacuees trapped in the room. And then, lower density represents higher evacuation efficiency and the more the information that evacuees receive the less the pedestrians dying during the evacuation process. At last, in respect of room structures, distributions (A) and (B) always perform better than distribution (C) no matter in aspects of death toll or evacuation efficiency. Therefore, administrator should choose the right area to place the hazardous article before the accident occurs and decision maker should choose the appropriate model parameters according to the actual condition to guide the evacuation when emergency happens. Further research works will be continued and focus on considering congestion management which could decrease the evacuation time and increase the usage rate of each exit.

\section{Conflicts of Interest}

The authors declare that they have no conflicts of interest.

\section{Acknowledgments}

The research work is partially supported by the National Natural Science Foundation of China (no. 71573190 and no. 71273188) and the Fundamental Research Funds for the Central Universities.

\section{References}

[1] J. Radianti, O.-C. Granmo, N. Bouhmala, P. Sarshar, A. Yazidi, and J. Gonzalez, "Crowd models for emergency evacuation: a review targeting human-centered sensing," in Proceedings of the 46th Annual Hawaii International Conference on System Sciences (HICSS '13), pp. 156-165, IEEE, Wailea, Hawaii, USA, January 2013.

[2] N. Li and Y. Xu, "Evacuation modeling from the control perspective and corresponding sequential-based optimal evacuation guidance," IEEE Transactions on Control Systems Technology, vol. 22, no. 3, pp. 1094-1102, 2014.

[3] N. Bellomo, B. Piccoli, and A. Tosin, "Modeling crowd dynamics from a complex system viewpoint," Mathematical Models and Methods in Applied Sciences, vol. 22, no. 2, Article ID 1230004, 29 pages, 2012.

[4] L. F. Henderson, "The statistics of crowd fluids," Nature, vol. 229, no. 5284, pp. 381-383, 1971.

[5] R. L. Hughes, "A continuum theory for the flow of pedestrians," Transportation Research Part B: Methodological, vol. 36, no. 6, pp. 507-535, 2002.

[6] X. Zheng, T. Zhong, and M. Liu, "Modeling crowd evacuation of a building based on seven methodological approaches," Building and Environment, vol. 44, no. 3, pp. 437-445, 2009. 
[7] D. Helbing, I. Farkas, and T. Vicsek, "Simulating dynamical features of escape panic," Nature, vol. 407, no. 6803, pp. 487490, 2000.

[8] Y. Qu, Z. Gao, Y. Xiao, and X. Li, "Modeling the pedestrian's movement and simulating evacuation dynamics on stairs," Safety Science, vol. 70, pp. 189-201, 2014.

[9] M. F. Li, Y. X. Zhao, L. Y. He, W. X. Chen, and X. F. Xu, “The parameter calibration and optimization of social force model for the real-life 2013 Yaan earthquake evacuation in China," Safety Science, vol. 79, pp. 243-253, 2015.

[10] C. Burstedde, K. Klauck, A. Schadschneider, and J. Zittartz, "Simulation of pedestrian dynamics using a two-dimensional cellular automaton," Physica A: Statistical Mechanics and Its Applications, vol. 295, no. 3-4, pp. 507-525, 2001.

[11] D.-F. Xie, Z.-Y. Gao, X.-M. Zhao, and D. Z.-W. Wang, "Agitated behavior and elastic characteristics of pedestrians in an alternative floor field model for pedestrian dynamics," Physica A: Statistical Mechanics and Its Applications, vol. 391, no. 7, pp. 2390-2400, 2012.

[12] J. Tanimoto, A. Hagishima, and Y. Tanaka, "Study of bottleneck effect at an emergency evacuation exit using cellular automata model, mean field approximation analysis, and game theory," Physica A: Statistical Mechanics and Its Applications, vol. 389, no. 24, pp. 5611-5618, 2010.

[13] Y.-C. Peng and C.-I. Chou, "Simulation of pedestrian flow through a 'T' intersection: a multi-floor field cellular automata approach," Computer Physics Communications, vol. 182, no. 1, pp. 205-208, 2011.

[14] J. Hu, H. Sun, G. Gao, J. Wei, and L. You, “The group evacuation behavior based on fire effect in the complicated threedimensional space," Mathematical Problems in Engineering, vol. 2014, Article ID 949280, 7 pages, 2014.

[15] S. Li, X. Li, Y. Qu, and B. Jia, "Block-based floor field model for pedestrian's walking through corner," Physica A: Statistical Mechanics and Its Applications, vol. 432, pp. 337-353, 2015.

[16] A. Kirchner, K. Nishinari, and A. Schadschneider, "Friction effects and clogging in a cellular automaton model for pedestrian dynamics," Physical Review E, vol. 67, no. 5, Article ID 056122, 2003.

[17] W. F. Yuan and K. H. Tan, "An evacuation model using cellular automata," Physica A: Statistical Mechanics and Its Applications, vol. 384, no. 2, pp. 549-566, 2007.

[18] T. Huan-Huan, D. Li-Yun, and X. Yu, "Influence of the exits' configuration on evacuation process in a room without obstacle," Physica A: Statistical Mechanics and Its Applications, vol. 420, pp. 164-178, 2015.

[19] W. Guo, X. Wang, M. Liu, Y. Cheng, and X. Zheng, "Modification of the dynamic floor field model by the heterogeneous bosons," Physica A: Statistical Mechanics and Its Applications, vol. 417, pp. 358-366, 2015.

[20] D. Hartmann and P. Hasel, "Efficient dynamic floor field methods for microscopic pedestrian crowd simulations," Communications in Computational Physics, vol. 16, no. 1, pp. 264286, 2014.

[21] Y. Zhao and Y. Z. Li, "Inertia effects on strategy updating in emergency evacuation from a room with multiple exits," International Journal of Modern Physics C, vol. 25, no. 9, Article ID 1450043, 2014.

[22] L.-L. Lu, G. Ren, W. Wang, and Y. Wang, "Modeling walking behavior of pedestrian groups with floor field cellular automaton approach," Chinese Physics B, vol. 23, no. 8, Article ID 088901, 2014.
[23] G. Yong, H.-J. Huang, and Y. Xu, "A cellular automata model of pedestrian evacuation in rooms with squared rhombus cells," Acta Physica Sinica, vol. 62, no. 1, Article ID 010506, 2013.

[24] S. Maniccam, “Traffic jamming on hexagonal lattice," Physica A. Statistical Mechanics and Its Applications, vol. 321, no. 3-4, pp. 653-664, 2003.

[25] B. Leng, J. Wang, W. Zhao, and Z. Xiong, "An extended floor field model based on regular hexagonal cells for pedestrian simulation," Physica A: Statistical Mechanics and Its Applications, vol. 402, pp. 119-133, 2014.

[26] Y. Zheng, B. Jia, X.-G. Li, and N. Zhu, "Evacuation dynamics with fire spreading based on cellular automaton," Physica A: Statistical Mechanics and Its Applications, vol. 390, no. 18-19, pp. 3147-3156, 2011.

[27] Y. Zheng, B. Jia, X. Li, and R. Jiang, "Evacuation dynamics considering pedestrians' movement behavior change with fire and smoke spreading," Safety Science, vol. 92, pp. 180-189, 2017.

[28] C. Wang, J. Wang, and Q. Bao, 2014 IEEE International Conference on Man and Cybernetics (SMC), San Diego, USA, Date 5-8 Oct. 2014, 2014.

[29] C. Wang and J. Wang, "A modified floor field model combined with risk field for pedestrian simulation," Mathematical Problems in Engineering, vol. 2016, Article ID 9653860, 10 pages, 2016.

[30] E. Ben-Jacob, "From snowflake formation to growth of bacterial colonies II: cooperative formation of complex colonial patterns," Contemporary Physics, vol. 38, no. 3, pp. 205-241, 1997.

[31] A. Kirchner and A. Schadschneider, "Simulation of evacuation processes using a bionics-inspired cellular automaton model for pedestrian dynamics," Physica A: Statistical Mechanics and Its Applications, vol. 312, no. 1-2, pp. 260-276, 2002.

[32] Z. Han, W. Weng, Q. Zhao, X. Ma, Q. Liu, and Q. Huang, "Investigation on an integrated evacuation route planning method based on real-time data acquisition for high-rise building fire," IEEE Transactions on Intelligent Transportation Systems, vol. 14, no. 2, pp. 782-795, 2013.

[33] Q. Li and D. Rus, "Navigation protocols in sensor networks," ACM Transactions on Sensor Networks, vol. 1, pp. 3-35, 2005. 


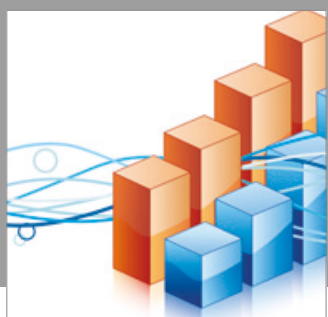

Advances in

Operations Research

vatersals

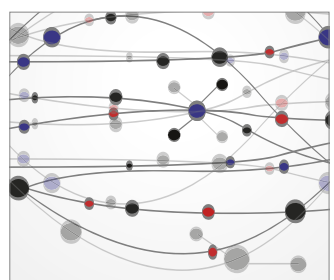

\section{The Scientific} World Journal
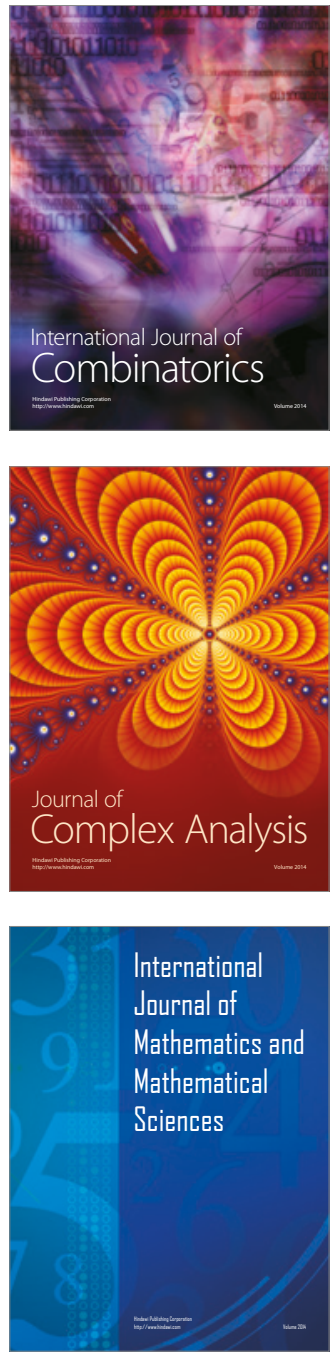
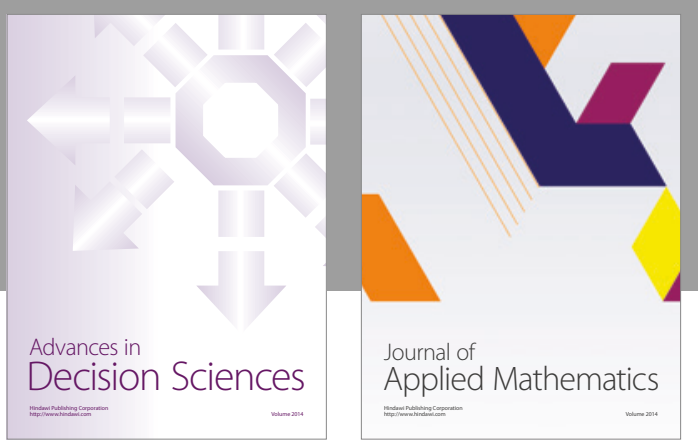

Algebra

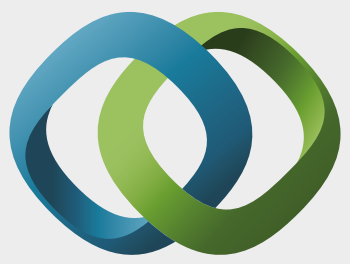

\section{Hindawi}

Submit your manuscripts at

https://www.hindawi.com
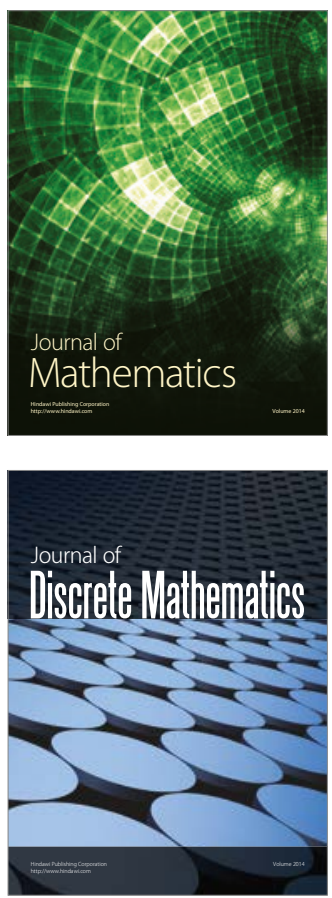

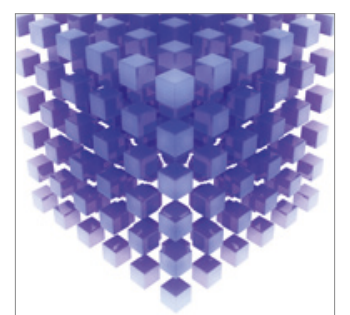

Mathematical Problems in Engineering
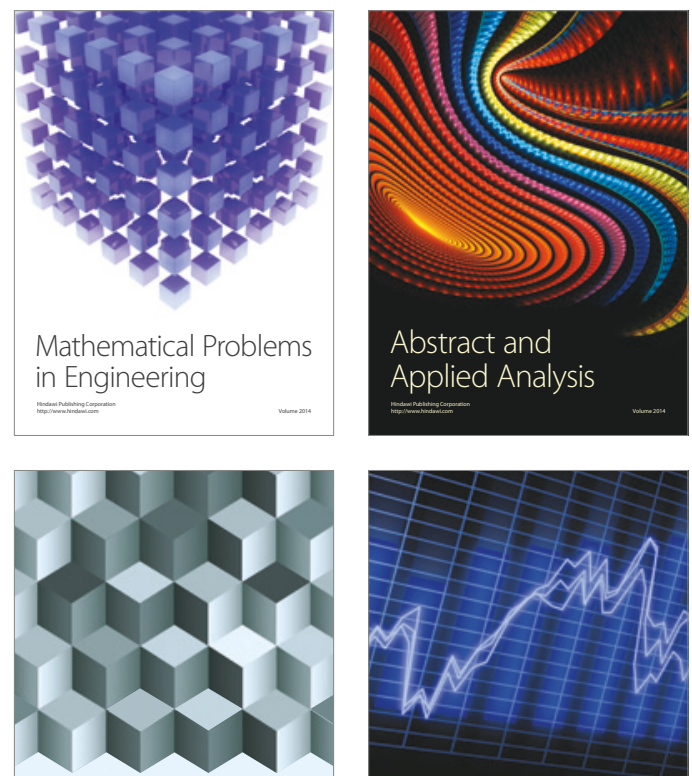

Journal of

Function Spaces

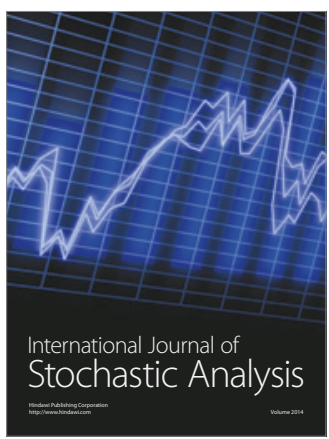

Probability and Statistics
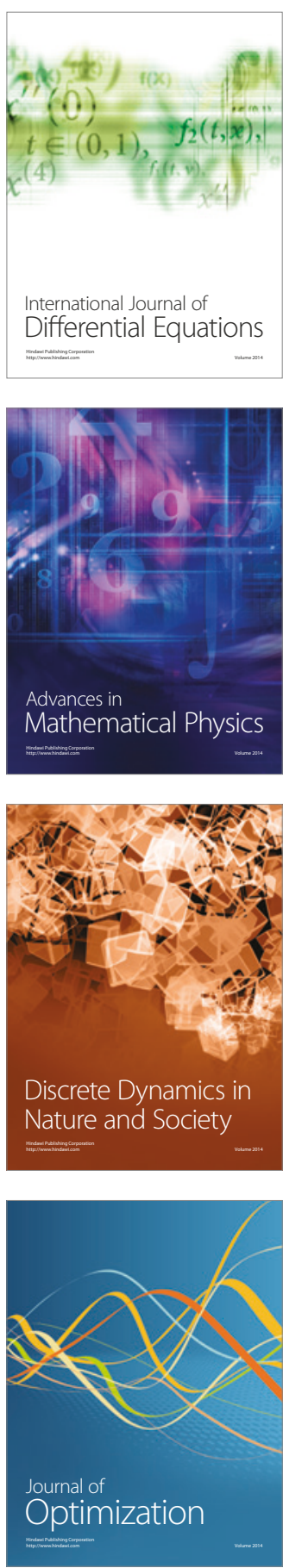